\title{
COTANGENT SPACES AND SEPARATING RE-EMBEDDINGS
}

\author{
MARTIN KREUZER, LE NGOC LONG, AND LORENZO ROBBIANO
}

\begin{abstract}
Given an affine algebra $R=P / I$, where $P=K\left[x_{1}, \ldots, x_{n}\right]$ is a polynomial ring over a field $K$ and $I$ is an ideal in $P$, we study re-embeddings of the affine scheme $\operatorname{Spec}(R)$, i.e., presentations $R \cong P^{\prime} / I^{\prime}$ such that $P^{\prime}$ is a polynomial ring in fewer indeterminates. To find such re-embeddings, we use polynomials $f_{i}$ in the ideal $I$ which are coherently separating in the sense that they are of the form $f_{i}=z_{i}-g_{i}$ with an indeterminate $z_{i}$ which divides neither a term in the support of $g_{i}$ nor in the support of $f_{j}$ for $j \neq i$. The possible numbers of such sets of polynomials are shown to be governed by the Gröbner fan of $I$. The dimension of the cotangent space of $R$ at a $K$-linear maximal ideal is a lower bound for the embedding dimension, and if we find coherently separating polynomials corresponding to this bound, we know that we have determined the embedding dimension of $R$ and found an optimal re-embedding.
\end{abstract}

\section{INTRODUCTION}

Given an affine scheme $\mathbb{X}$ embedded in an affine space $\mathbb{A}_{K}^{n}$ over a field $K$, it is a natural question to ask whether $\mathbb{X}$ can be embedded into an affine space of lower dimension. For instance, a classical result in algebraic geometry says that a smooth affine variety of dimension $d$ over an infinite field $K$ can be embedded into $\mathbb{A}_{K}^{2 d+1}$. This was generalized by V. Srinivas to the non-smooth case in [17. The method to prove these results is to start with an embedding $\mathbb{X} \longrightarrow \mathbb{A}_{K}^{n}$ into a high-dimensional affine space and then to apply general linear projections which re-embed $\mathbb{X}$, i.e., which define isomorphisms on $\mathbb{X}$. Partial solutions to the above problem can be found in many papers. Among them, it is worth mentioning [2, 4, and 14 where an approach of algebraic nature is used which is, to a certain extent, similar to our method.

Although the general problem of finding the minimal number of algebra generators of an affine $K$-algebra seems to be very hard, it turns out to be possible to re-embed some affine schemes in much lower dimensional affine spaces using linear projections based on separating indeterminates. For instance, if a polynomial in the given ideal is of the form $f=z-g$ where the indeterminate $z$ does not divide any term in the support of $g$, one can eliminate $z$ from the polynomials in the given ideal in an obvious way. To eliminate several indeterminates at a time, one needs a coherently separating set of polynomials in the sense defined below. The main advantage of such re-embeddings is that they do not involve generic changes of coordinates. Here the possible dimensions of the ambient spaces after the reembeddings are controlled by the Gröbner fan of the given ideal which, alas, is hard to compute in general.

Can we reach the embedding dimension, i.e., the smallest dimension of the ambient space in this way? In general, this is impossible, because the optimal reembedding may not be achieved via linear projections. One immediate obstruction

2010 Mathematics Subject Classification. Primary 13P10; Secondary 14Q20, 13E15, 14R10.

Key words and phrases. cotangent space, embedding dimension, affine scheme, Gröbner basis Gröbner fan, border basis scheme. 
is the dimension of the tangent spaces at the $K$-rational points of the scheme. There can be no isomorphic re-embedding into a space which has a lower dimension than one of the tangent spaces. However, it is quite nice and useful to observe that if we reach this dimension at some point, the resulting re-embedding is optimal and we have found the embedding dimension. For instance, in the last section of the paper we show an example where our method allows us to compute an optimal embedding of a singular border basis scheme (see Example 4.6). More on good embeddings of border basis schemes will be discussed in a forthcoming paper.

To achieve these goals, we proceed as follows. In Section 1 we consider an affine algebra $R=P / I$, where $P=K\left[x_{1}, \ldots, x_{n}\right]$ is a polynomial ring over a field $K$ and $I$ is an ideal in $P$. We observe that the computation of the tangent and cotangent spaces of $R$ at a maximal ideal $\mathfrak{m}=\left\langle x_{1}-a_{1}, \ldots, x_{n}-a_{n}\right\rangle / I$ corresponding to a $K$-rational point $\left(a_{1}, \ldots, a_{n}\right)$ of $\mathbb{X}=\operatorname{Spec}(R)$ boils down to the calculation of the $\mathfrak{M}$-linear part of $I$, where $\mathfrak{M}=\left\langle x_{1}-a_{1}, \ldots, x_{n}-a_{n}\right\rangle \supseteq I$ (see Prop. 1.5), and can be achieved efficiently using any set of generators of $I$ (see Prop. 1.6).

In Section 2 we introduce the important concept of separating re-embeddings. Given a set of distinct indeterminates $Z=\left\{z_{1}, \ldots, z_{s}\right\}$ in $X=\left\{x_{1}, \ldots, x_{n}\right\}$, we say that a set of polynomials $f_{1}, \ldots, f_{s} \in I$ is coherently $Z$-separating if each $f_{i}$ is of the form $f_{i}=z_{i}-g_{i}$ with $g_{i} \in P$ such that $z_{i}$ divides neither a term in the support of $g_{i}$ nor in the support of $f_{j}$ with $j \neq i$. This property is equivalent to the existence of Gröbner bases of $I$ of a very special shape (see Prop. 2.6) which in turn allow us to define an isomorphism $\Phi: P / I \longrightarrow \widehat{P} /(I \cap \widehat{P})$, where $\widehat{P}=K[X \backslash Z]$ has fewer indeterminates (see Thm. 2.13). Clearly, the map $\Phi$ corresponds to a re-embedding of $\mathbb{X}=\operatorname{Spec}(R)$ into a lower-dimensional affine space. We call it the $Z$-separating re-embedding of $I$.

In Section 3 we study the possible choices of the sets of indeterminates $Z$ for which a coherently $Z$-separating set of polynomials exists in the given ideal $I$. Via the Gröbner bases mentioned above, they are related to the Gröbner fan of $I$, i.e., to the set of marked reduced Gröbner bases of $I$. Here the sets $Z$ correspond to the sets of leading indeterminates of a reduced Gröbner basis of $I$ (see Prop. 2.14). The existence of an optimal separating re-embedding of $I$ for which the ambient dimension equals the embedding dimension of $R$ is therefore equivalent to the existence of a reduced Gröbner basis of $I$ with enough leading terms which are indeterminates (see Prop. [3.6). This correspondence can be made 1-1 by introducing a suitable equivalence relation on the set of reduced Gröbner bases of $I$ (see Prop. [3.9) ).

Consequently, we search for optimal re-embeddings in Section 4. The key observation is that the dimension of the tangent space of $R$ at any $K$-rational point is a lower bound for the embedding dimension (see Thm. 4.1) and that we know we have reached the embedding dimension if we find one set of coherently $Z$-separating polynomials of the correct cardinality (see Cor. 4.2). Here the required cardinality of $Z$ can be calculated efficiently using the technique explained in Section 1. Suitable examples illustrate our main results.

The notation and definitions in this paper follow 8 and 9 . The calculations underlying most examples were performed using the computer algebra system [1].

\section{Tangent and Cotangent Spaces}

In this section we introduce some concepts which play a fundamental role in the paper. Most of them are well-known, however, for the sake of clarity and coherence of notation, we include them together with propositions, proofs, and examples.

Let $K$ be a field, let $P=K\left[x_{1}, \ldots, x_{n}\right]$, let $p=\left(a_{1}, \ldots, a_{n}\right) \in K^{n}$, and let $\mathfrak{M}$ be the linear maximal ideal $\mathfrak{M}=\left\langle x_{1}-a_{1}, \ldots, x_{n}-a_{n}\right\rangle$. In the following we assume 
that $I$ is an ideal in $P$ such that $I \subseteq \mathfrak{M}$. Then we let $R=P / I$, we consider the maximal ideal $\mathfrak{m}=\mathfrak{M} / I$ of $R$, and we write $\mathbb{X}=\operatorname{Spec}(R)$ for the affine scheme associated to $R$. Notice that $I \subseteq \mathfrak{M}$ implies $R / \mathfrak{m} \cong P / \mathfrak{M} \cong K$.

The first fundamental objects that we need in the following are the tangent space and the cotangent space of $\mathbb{X}$ at a point $p$. Let us recall them.

Definition 1.1. As above, let $p=\left(a_{1}, \ldots, a_{n}\right)$, let $\mathfrak{M}=\left\langle x_{1}-a_{1}, \ldots, x_{n}-a_{n}\right\rangle$, let $I$ be an ideal in $P$ which is contained in $\mathfrak{M}$, and let $\mathfrak{m}=\mathfrak{M} / I$.

(a) The $K$-vector space $\operatorname{Cot}_{\mathfrak{m}}(R)=\mathfrak{m} / \mathfrak{m}^{2}$ is called the Zariski cotangent space of $R$ at the point $p$. It is also denoted by $\operatorname{Cot}_{p}(\mathbb{X})$.

(b) The dual vector space $\operatorname{Tan}_{\mathfrak{m}}(R)=\operatorname{Hom}_{K}\left(\mathfrak{m} / \mathfrak{m}^{2}, K\right)$ of the cotangent space is called the Zariski tangent space of $R$ at the point $p$. It is also denoted by $\operatorname{Tan}_{p}(\mathbb{X})$.

In order to calculate tangent and cotangent spaces, it will be useful to change the coordinate system so that the point $p$ under consideration is the origin. The following notation will come in handy.

Notation 1.2. In the above setting, we introduce new indeterminates $y_{1}, \ldots, y_{n}$. Then we define the $K$-algebra isomorphism

$$
\varphi_{p}: P \longrightarrow K\left[y_{1}, \ldots, y_{n}\right] \quad \text { given by } x_{i} \mapsto y_{i}+a_{i} \text { for } i=1, \ldots, n
$$

and its inverse

$$
\psi_{p}: K\left[y_{1}, \ldots, y_{n}\right] \longrightarrow P \quad \text { given by } \quad y_{i} \mapsto x_{i}-a_{i} \text { for } i=1, \ldots, n .
$$

The homogeneous maximal ideal of $K\left[y_{1}, \ldots, y_{n}\right]$ is $\mathfrak{N}=\left\langle y_{1}, \ldots, y_{n}\right\rangle$. Clearly, we have $\mathfrak{N}=\varphi_{p}(\mathfrak{M})$. For every $i \geq 0$, its homogeneous component of degree $i$ is denoted by $\mathfrak{N}_{i}$. In particular, we have $\mathfrak{N}^{i}=\bigoplus_{j \geq i} \mathfrak{N}_{j}$ for every $i \geq 1$.

Now we are ready to introduce the following generalization of the linear part of a polynomial.

Definition 1.3. As above, let $I$ be an ideal in $P$ which is contained in the maximal ideal $\mathfrak{M}=\left\langle x_{1}-a_{1}, \ldots, x_{n}-a_{n}\right\rangle$.

(a) Given $f \in P$, let $\ell \in \mathfrak{N}_{1}$ be the homogeneous component of degree one of $\varphi_{p}(f)=f\left(y_{1}+a_{1}, \ldots, y_{n}+a_{n}\right)$. Then the polynomial $\operatorname{Lin}_{\mathfrak{M}}(f)=\psi_{p}(\ell)=$ $\ell\left(x_{1}-a_{1}, \ldots, x_{n}-a_{n}\right)$ is called the $\mathfrak{M}$-linear part of $f$.

(b) The $K$-vector space $\operatorname{Lin}_{\mathfrak{M}}(I)=\left\langle\operatorname{Lin}_{\mathfrak{M}}(f) \mid f \in I\right\rangle_{K}$ is called the $\mathfrak{M}$-linear part of $I$.

Notice that the result $\varphi_{p}(f)$ of the substitution in part (a) of this definition has no constant term if and only if $f \in \mathfrak{M}$. Clearly, the definition yields an effective method for computing $\operatorname{Lin}_{\mathfrak{M}}(f)$. The following example illustrates it.

Example 1.4. Let $P=\mathbb{Q}\left[x_{1}, x_{2}\right]$, let $\mathfrak{M}=\left\langle x_{1}-1, x_{2}-2\right\rangle$, and let $f=x_{1}^{2}+$ $x_{2}^{2}-x_{1}-4$. Then we calculate $g=f\left(y_{1}+1, y_{2}+2\right)=y_{1}^{2}+y_{2}^{2}+y_{1}+4 y_{2}$ and $\operatorname{Lin}_{\mathfrak{N}}(g)=y_{1}+4 y_{2}$. Hence we obtain $\operatorname{Lin}_{\mathfrak{M}}(f)=\left(x_{1}-1\right)+4\left(x_{2}-2\right)=x_{1}+4 x_{2}-9$.

Now the cotangent and the tangent space of $\mathbb{X}=\operatorname{Spec}(R)$ at $p$ can be described as follows.

Proposition 1.5. As in the above setting, let $p=\left(a_{1}, \ldots, a_{n}\right) \in K^{n}$, let $\mathfrak{M}=$ $\left\langle x_{1}-a_{1}, \ldots, x_{n}-a_{n}\right\rangle$, and let $I$ be an ideal in $P$ which is contained in $\mathfrak{M}$.

(a) Let $\mathfrak{M}_{1}=\left\langle x_{1}-a_{1}, \ldots, x_{n}-a_{n}\right\rangle_{K}$. Then we have $\operatorname{Lin}_{\mathfrak{M}}(I)=\mathfrak{M}_{1} \cap\left(I+\mathfrak{M}^{2}\right)$.

(b) There is an isomorphism of $K$-vector spaces $\operatorname{Cot}_{\mathfrak{m}}(R) \cong \mathfrak{M}_{1} / \operatorname{Lin}_{\mathfrak{M}}(I)$.

(c) There is an isomorphism of $K$-vector spaces

$$
\operatorname{Tan}_{\mathfrak{m}}(R) \cong\left\{v \in K^{n} \mid \ell(p+v)=0 \text { for all } \ell \in \operatorname{Lin}_{\mathfrak{M}}(I)\right\}
$$


Proof. To prove (a), we let $J=\varphi_{p}(I)$. By the definition of the linear part of an ideal, we have to show $\operatorname{Lin}_{\mathfrak{N}}(J)=\mathfrak{N}_{1} \cap\left(J+\mathfrak{N}^{2}\right)$, where we know that $J \subseteq \mathfrak{N}$. Since the inclusion $\subseteq$ is trivially true, it suffices to prove $\supseteq$. Let $\ell \in \mathfrak{N}_{1}$ be of the form $\ell=f+g$ with $f \in J$ and $g \in \mathfrak{N}^{2}$. Then the equality $f=\ell-g$ shows that $\ell=\operatorname{Lin}_{\mathfrak{N}}(f)$, and hence $\ell \in \operatorname{Lin}_{\mathfrak{N}}(J)$, as claimed.

To prove (b), we note that $\operatorname{Cot}_{\mathfrak{m}}(R)=\mathfrak{m} / \mathfrak{m}^{2} \cong \mathfrak{M} /\left(I+\mathfrak{M}^{2}\right)$. Using $\mathfrak{N}^{i}=$ $\bigoplus_{j \geq i} \mathfrak{N}_{j}$ for $i \geq 1$, the isomorphism $\psi_{p}$ yields direct sum decompositions $\mathfrak{M}=$ $\bigoplus_{i>1} \mathfrak{M}_{i}$ and $\mathfrak{M}^{2}=\bigoplus_{i>2} \mathfrak{M}_{i}$, where $\mathfrak{M}_{i}=\psi_{p}\left(\mathfrak{N}_{i}\right)$ for $i \geq 1$. Hence we get that $\operatorname{Cot}_{\mathfrak{m}}(R)$ is the residue class vector space of $\mathfrak{M}_{1}$ modulo $\mathfrak{M}_{1} \cap\left(I+\mathfrak{M}^{2}\right)$, and the latter intersection equals $\operatorname{Lin}_{\mathfrak{M}}(I)$ by (a).

Claim (c) is a consequence of (b) and the observation that a linear form $\ell=$ $c_{1}\left(x_{1}-a_{1}\right)^{*}+\cdots+c_{n}\left(x_{n}-a_{n}\right)^{*}$ in the dual vector space of $\mathfrak{M}_{1}$ is exactly the evaluation at $p+\left(c_{1}, \ldots, c_{n}\right) \in K^{n}$.

In view of this proposition, the tasks to compute the Zariski cotangent and tangent spaces of $R$ at $p$ are reduced to computing the $\mathfrak{M}$-linear part of $I$. This can be done as follows.

Proposition 1.6. As in the above setting, let $p=\left(a_{1}, \ldots, a_{n}\right) \in K^{n}$, let $\mathfrak{M}=$ $\left\langle x_{1}-a_{1}, \ldots, x_{n}-a_{n}\right\rangle$, let $f_{1}, \ldots, f_{s} \in \mathfrak{M}$, and let $I=\left\langle f_{1}, \ldots, f_{s}\right\rangle$. Then we have

$$
\operatorname{Lin}_{\mathfrak{M}}(I)=\left\langle\operatorname{Lin}_{\mathfrak{M}}\left(f_{1}\right), \ldots, \operatorname{Lin}_{\mathfrak{M}}\left(f_{s}\right)\right\rangle_{K}
$$

Proof. For $i=1, \ldots, s$, we write $f_{i}=\ell_{i}+g_{i}$ with $\ell_{i}=\operatorname{Lin}_{\mathfrak{M}}\left(f_{i}\right)$ and $g_{i} \in \mathfrak{M}^{2}$. Then we have $I+\mathfrak{M}^{2}=\left\langle\ell_{1}, \ldots, \ell_{s}\right\rangle+\mathfrak{M}^{2}$ and therefore, using Proposition 1.5 a, we get $\operatorname{Lin}_{\mathfrak{M}}(I)=\mathfrak{M}_{1} \cap\left(I+\mathfrak{M}^{2}\right)=\mathfrak{M}_{1} \cap\left(\left\langle\ell_{1}, \ldots, \ell_{s}\right\rangle+\mathfrak{M}^{2}\right)$. When we apply the isomorphism $\varphi_{p}$, we see that the only homogeneous polynomials of degree one in the image of the ideal $\left\langle\ell_{1}, \ldots, \ell_{s}\right\rangle+\mathfrak{M}^{2}$ are the ones in the image of the vector space $\left\langle\ell_{1}, \ldots, \ell_{s}\right\rangle_{K}$. Thus we get $\operatorname{Lin}_{\mathfrak{M}}(I)=\left\langle\ell_{1}, \ldots, \ell_{s}\right\rangle_{K}$, as claimed.

Notice that this proposition implies that Zariski tangent and cotangent spaces can be computed efficiently using any system of generators of $I$. If the ideal $I$ is not contained in $\mathfrak{M}$, the conclusions of the proposition may fail, as the following example shows.

Example 1.7. Let $P=\mathbb{Q}[x, y, z]$, let $\mathfrak{M}=\langle x, y, z\rangle$, and let $I=\left\langle f_{1}, f_{2}\right\rangle$, where $f_{1}=x+1$ and $f_{2}=y-z-z^{2}$. Then $g=y f_{1}-x f_{2}=x z^{2}+x z+y \in I$ implies that $y=\operatorname{Lin}_{\mathfrak{M}}(g)$ is in the $\mathfrak{M}$-linear part of $I$. However, $y$ is not contained in the ideal $\left\langle\operatorname{Lin}_{\mathfrak{M}}\left(f_{1}\right), \operatorname{Lin}_{\mathfrak{M}}\left(f_{2}\right)\right\rangle=\langle x, y-z\rangle$.

Let us use the above results to compute some cotangent and tangent spaces.

Example 1.8. Let $P=\mathbb{Q}[x, y, z]$, let $\mathfrak{M}=\langle x, y, z\rangle$, and let $I=\left\langle f_{1}, f_{2}, f_{3}\right\rangle$, where $f_{1}=x^{3}-x-z, f_{2}=y^{2}+x$, and $f_{3}=x y+z$. Then we get $\operatorname{Lin}_{\mathfrak{M}}(I)=$ $\left\langle\operatorname{Lin}_{\mathfrak{M}}\left(f_{1}\right), \operatorname{Lin}_{\mathfrak{M}}\left(f_{2}\right), \operatorname{Lin}_{\mathfrak{M}}\left(f_{3}\right)\right\rangle=\langle-x-z, x, z\rangle=\langle x, z\rangle$. Hence the cotangent space of $R=P / I$ at $p=(0,0,0)$ is $\operatorname{Cot}_{\mathfrak{m}}(R)=\mathfrak{m} / \mathfrak{m}^{2} \cong\langle x, y, z\rangle /\left\langle x, z, y^{2}\right\rangle \cong K \cdot \bar{y}$ and the tangent space is $\operatorname{Tan}_{\mathfrak{m}}(R)=K \cdot(0,1,0)$.

Example 1.9. Let $P=\mathbb{Q}[x, y, z]$, let $\mathfrak{M}=\langle x, y, z\rangle$, and let $I=\left\langle f_{1}, f_{2}\right\rangle$, where $f_{1}=x^{3} z-x$ and $f_{2}=y z^{2}-x$. Then we have $\operatorname{Lin}_{\mathfrak{M}}(I)=\langle x\rangle$. Therefore the cotangent space of $R=P / I$ at the origin is the 2-dimensional vector space $\operatorname{Cot}_{\mathfrak{m}}(R)=\mathfrak{m} / \mathfrak{m}^{2} \cong\langle x, y, z\rangle /\left\langle x, y^{2}, y z, z^{2}\right\rangle \cong K \cdot \bar{y} \oplus K \cdot \bar{z}$, and the tangent space is $\operatorname{Tan}_{\mathfrak{m}}(R)=K \cdot(0,1,0) \oplus K \cdot(0,0,1)$. 


\section{Separating Re-EMbeddings}

In this section we continue to study affine $K$-algebras $R=P / I$, where $P=$ $K\left[x_{1}, \ldots, x_{n}\right]$ is a polynomial ring over a field $K$ and $I$ is an ideal in $P$. The presentation $R=P / I$ corresponds to an embedding of the affine scheme $\mathbb{X}=$ $\operatorname{Spec}(R)$ into the affine $n$-space $\mathbb{A}_{K}^{n}$. Computing another presentation $R \cong P^{\prime} / I^{\prime}$ with $P^{\prime}=K\left[y_{1}, \ldots, y_{m}\right]$ and an ideal $I^{\prime}$ in $P^{\prime}$ amounts to embedding $\mathbb{X}$ into another affine space, preferably of lower dimension $m<n$.

Starting from this point, we assume that the linear maximal ideal under consideration is $\mathfrak{M}=\left\langle x_{1}, \ldots, x_{n}\right\rangle$. We leave it to the interested reader to generalize the subsequent results to arbitrary linear maximal ideals of $P$ with the help of Proposition 1.6. Our goal is to construct alternative embeddings of $\mathbb{X}$ using particular generators of the ideal $I$. The main ideas and results of this section can be viewed as part of elimination theory which is explained in great detail for instance in Section 3.4 of [8] (see Remark 2.7). The next definition provides a key construction.

Definition 2.1. In the above setting, let $\mathfrak{M}=\left\langle x_{1}, \ldots, x_{n}\right\rangle$, and let $f \in \mathfrak{M}$.

(a) The set of all indeterminates in $\left\{x_{1}, \ldots, x_{n}\right\}$ which divide at least one of the terms in the support of $f$ is called the set of indeterminates of $f$ and is denoted by indets $(f)$.

(b) Assuming $\operatorname{Lin}_{\mathfrak{M}}(f) \neq 0$, let $z \in \operatorname{indets}\left(\operatorname{Lin}_{\mathfrak{M}}(f)\right)$, and let $c \in K \backslash\{0\}$ be the coefficient of $z$ in $f$. Then the polynomial $z-\frac{1}{c} f$ is called the $z$-tail of $f$ and is denoted by $\operatorname{tail}_{z}(f)$.

(c) For every indeterminate $z \in \operatorname{indets}\left(\operatorname{Lin}_{\mathfrak{M}}(f)\right)$ such that $z \notin \operatorname{indets}\left(\operatorname{tail}_{z}(f)\right)$ we say that $f$ is $z$-separating.

The notion of being $z$-separating can be characterized as follows.

Remark 2.2. Let $f \in \mathfrak{M}$ with $\operatorname{Lin}_{\mathfrak{M}}(f) \neq 0$, and let $z \in \operatorname{indets}\left(\operatorname{Lin}_{\mathfrak{M}}(f)\right)$. Then $f$ is $z$-separating if and only if there exists a term ordering $\sigma$ such that $z=\operatorname{LT}_{\sigma}(f)$. In this case the reduced $\sigma$-Gröbner basis of the principal ideal $\langle f\rangle$ is $\left\{\frac{1}{c} f\right\}$.

Note that $\frac{1}{c} f=z-\operatorname{tail}_{z}(f)$ and that $\operatorname{tail}_{z}(f) \in \widehat{P}$, where $\widehat{P}$ is the polynomial ring in the indeterminates $\left\{x_{1}, \ldots, x_{n}\right\} \backslash\{z\}$ over $K$. Consequently, the substitution $z \mapsto \operatorname{tail}_{z}(f)$ yields a $K$-algebra isomorphism $P /\langle f\rangle \cong \widehat{P}$ which allows us to identify the hypersurface $\operatorname{Spec}(P /\langle f\rangle)$ in $\mathbb{A}_{K}^{n}$ with the affine space $\mathbb{A}_{K}^{n-1}$.

The following example indicates a way how to use a separating polynomial in order to find embeddings into affine spaces of lower dimension.

Example 2.3. Let $P=\mathbb{Q}[x, y, z]$, and let $I=\langle f, g\rangle$, where $f=y^{3} z-z^{4}+2 x$ and $g=z^{2}-x y-y$. Clearly, the polynomial $f$ is $x$-separating. Let $\sigma$ be the elimination ordering $\operatorname{Elim}(x)$ (see [8], Def. 1.4.10). Then the reduced $\sigma$-Gröbner basis of $I$ is

$$
\left\{\frac{1}{2} f, y^{4} z-y z^{4}+2 z^{2}-2 y\right\}=\left\{x-\left(-\frac{1}{2} y^{3} z+\frac{1}{2} z^{4}\right), y^{4} z-y z^{4}+2 z^{2}-2 y\right\}
$$

Hence [8], Thm. 3.4.5, yields $I \cap \mathbb{Q}[y, z]=\left\langle y^{4} z-y z^{4}+2 z^{2}-2 y\right\rangle$, and the substitution $x \mapsto-\frac{1}{2} y^{3} z+\frac{1}{2} z^{4}$ induces a $\mathbb{Q}$-algebra isomorphism

$$
P / I \cong \mathbb{Q}[y, z] /\left\langle y^{4} z-y z^{4}+2 z^{2}-2 y\right\rangle
$$

Geometrically, we have embedded the subscheme $\operatorname{Spec}(P / I)$ of $\mathbb{A}_{\mathbb{Q}}^{3}$ into $\mathbb{A}_{\mathbb{Q}}^{2}$.

This example motivates us to try to eliminate several indeterminates simultaneously. To keep the notation manageable, we introduce the following setting.

Notation 2.4. The set of indeterminates of $P$ is denoted by $X=\left\{x_{1}, \ldots, x_{n}\right\}$. Let $s \geq 1$. For $i=1, \ldots, s$, we choose pairwise distinct indeterminates $z_{i} \in X$, 
and we let $Z$ be the set $Z=\left\{z_{1}, \ldots, z_{s}\right\}$. Moreover, the remaining indeterminates $Y=X \backslash Z$ will be written as $Y=\left\{y_{1}, \ldots, y_{n-s}\right\}$, and we denote the polynomial ring in these indeterminates by $\widehat{P}=K\left[y_{1}, \ldots, y_{n-s}\right]$. Furthermore, given a term ordering $\sigma$ on $P$, its restriction to $\widehat{P}$ is denoted by $\hat{\sigma}$.

The following definition provides a condition for the simultaneous elimination of several indeterminates to work.

Definition 2.5. Let $s \geq 1$, let $f_{1}, \ldots, f_{s} \in \mathfrak{M} \backslash\{0\}$, and let $Z=\left\{z_{1}, \ldots, z_{s}\right\}$ be a set of $s$ distinct indeterminates in $X$. Then we say that the tuple $\left(f_{1}, \ldots, f_{s}\right)$ is coherently $Z$-separating if the following two conditions are satisfied for every $i \in\{1, \ldots, s\}$.

(1) The polynomial $f_{i}$ is $z_{i}$-separating.

(2) For $j \neq i$, we have $z_{i} \notin \operatorname{indets}\left(f_{j}\right)$.

Generalizing Remark 2.2, we can characterize coherently $Z$-separating tuples of polynomials as follows.

Proposition 2.6. Let $s \geq 1$, let $f_{1}, \ldots, f_{s} \in \mathfrak{M} \backslash\{0\}$, and let $Z=\left\{z_{1}, \ldots, z_{s}\right\}$ be a set of $s$ distinct indeterminates in $X$. Then the following conditions are equivalent.

(a) The tuple $\left(f_{1}, \ldots, f_{s}\right)$ is coherently $Z$-separating.

(b) For every term ordering $\sigma$ such that $z_{i}=\operatorname{LT}_{\sigma}\left(f_{i}\right)$ for $i=1, \ldots, s$, the reduced $\sigma$-Gröbner basis of $\left\langle f_{1}, \ldots, f_{s}\right\rangle$ is of the form $\left\{\frac{1}{c_{1}} f_{1}, \ldots, \frac{1}{c_{s}} f_{s}\right\}$, where $c_{i}=\mathrm{LC}_{\sigma}\left(f_{i}\right)$ for $i=1, \ldots, s$.

(c) For every proper ideal $I$ in $P$ containing $f_{1}, \ldots, f_{s}$ and for every term ordering $\sigma$ such that $z_{i}=\mathrm{LT}_{\sigma}\left(f_{i}\right)$ for $i=1, \ldots, s$, the ideal $I$ has a $\sigma$ Gröbner basis of the form $\left\{\frac{1}{c_{1}} f_{1}, \ldots, \frac{1}{c_{s}} f_{s}, g_{1}, \ldots, g_{t}\right\}$, where $c_{i}=\mathrm{LC}_{\sigma}\left(f_{i}\right)$ for $i=1, \ldots, s$, where the reduced $\sigma$-Gröbner basis of $\left\langle f_{1}, \ldots, f_{s}\right\rangle$ is $\left\{\frac{1}{c_{1}} f_{1}, \ldots, \frac{1}{c_{s}} f_{s}\right\}$, and where $\left\{g_{1}, \ldots, g_{t}\right\}$ is the reduced $\hat{\sigma}-$ Gröbner basis of $I \cap \widehat{P}$.

Proof. First we show that (a) implies (b). Let $\sigma$ be a term ordering such that $\operatorname{LT}_{\sigma}\left(f_{i}\right)=z_{i}$ for $i=1, \ldots, s$. Then the hypothesis implies that $z_{i}$ does not divide any term in the support of $\operatorname{tail}_{z_{i}}\left(f_{i}\right)$ or in the support of one of the polynomials $f_{j}$ with $j \neq i$. Since the leading terms $z_{i}=\operatorname{LT}_{\sigma}\left(f_{i}\right)$ are pairwise coprime, the set $\left\{\frac{1}{c_{1}} f_{1}, \ldots, \frac{1}{c_{s}} f_{s}\right\}$ is a $\sigma$-Gröbner basis of $\left\langle f_{1}, \ldots, f_{s}\right\rangle$. Using $z_{i} \notin \operatorname{indets}\left(f_{j}\right)$ for $i \neq j$, we see that this set is fully interreduced, and therefore it is the reduced $\sigma$-Gröbner basis of $\left\langle f_{1}, \ldots, f_{s}\right\rangle$.

Next we show that (b) implies (c). Using $z_{i}=\operatorname{LT}_{\sigma}\left(f_{i}\right)$ for $i=1, \ldots, s$ and the hypothesis that $I$ is not the unit ideal in $P$, it follows that the indeterminates $z_{1}, \ldots, z_{s}$ are part of a minimal system of generators of $\operatorname{LT}_{\sigma}(I)$. Hence the set $\left\{\frac{1}{c_{1}} f_{1}, \ldots, \frac{1}{c_{s}} f_{s}\right\}$ is part of a minimal $\sigma$-Gröbner basis of $I$. Let $\left\{\tilde{g}_{1}, \ldots, \tilde{g}_{t}\right\}$ be the remaining elements of this minimal Gröbner basis. As the leading terms $\operatorname{LT}_{\sigma}\left(\tilde{g}_{i}\right)$ are not divisible by any indeterminate in $Z$, we can replace $\tilde{g}_{i}$ by their normal remainders $\hat{g}_{i}$ with respect to division by $\left(f_{1}, \ldots, f_{s}\right)$. Then we have $\hat{g}_{i} \in \widehat{P}$ for $i=1, \ldots, t$. Finally, we can divide the polynomials $\hat{g}_{i}$ by their leading coefficients and interreduce them to get the reduced $\hat{\sigma}$-Gröbner basis $\left\{g_{1}, \ldots, g_{t}\right\}$ of $I \cap \widehat{P}$.

The implication $(\mathrm{c}) \Rightarrow(\mathrm{b})$ is clear, and the implication $(\mathrm{b}) \Rightarrow(\mathrm{a})$ follows directly from the definition of a reduced Gröbner basis. Thus the proof is complete.

The following remark says that suitable term orderings always exist.

Remark 2.7. Notice that, for a coherently $Z$-separating tuple $\left(f_{1}, \ldots, f_{s}\right)$, any elimination ordering $\sigma$ for $Z$ has the property $\operatorname{LT}_{\sigma}\left(f_{i}\right)=z_{i}$ required by conditions (b) and (c) of the proposition. 
The next example illustrates the proposition.

Example 2.8. Let $P=\mathbb{Q}[x, y, z]$, and let $I=\left\langle f_{1}, f_{2}\right\rangle$, where $f_{1}=x^{2}-x-y$ and $f_{2}=y^{2}-z$.

(a) The polynomial $f_{1}$ is $y$-separating and $f_{2}$ is $z$-separating. However, the tuple $\left(f_{1}, f_{2}\right)$ is not coherently $Z$-separating with respect to $Z=\{y, z\}$, since $y$ appears in the $z$-tail of $f_{2}$.

(b) Let $\sigma$ be the term ordering $\operatorname{Elim}(y, z)$. Then the reduced $\sigma$-Gröbner basis of $I$ is $\left\{-f_{1}, g\right\}$, where $g=z-\left(x^{2}-x\right)^{2}$. Clearly, the tuple $\left(f_{1}, g\right)$ is coherently $Z$-separating with respect to $Z=\{y, z\}$.

Proposition 2.6 suggests the following definition.

Definition 2.9. Let $s \geq 1$, let $Z=\left\{z_{1}, \ldots, z_{s}\right\}$ be a set of $s$ distinct indeterminates in $X$, and let $I \subseteq \mathfrak{M}$ be an ideal.

(a) A term ordering $\sigma$ on $\mathbb{T}^{n}$ is called a $Z$-separating term ordering for $I$ if there exist polynomials $f_{1}, \ldots, f_{s} \in I \backslash\{0\}$ such that $z_{i}=\operatorname{LT}_{\sigma}\left(f_{i}\right)$ for $i=1, \ldots, s$ and $\left(f_{1}, \ldots, f_{s}\right)$ is coherently $Z$-separating.

(b) Let $\sigma$ be a $Z$-separating term ordering for $I$ on $\mathbb{T}^{n}$. Then a $\sigma$-Gröbner basis of $I$ is called a $Z$-separating $\sigma$-Gröbner basis of $I$ if it is of the form $\left\{\frac{1}{c_{1}} f_{1}, \ldots, \frac{1}{c_{s}} f_{s}, g_{1}, \ldots, g_{t}\right\}$ where $\left(f_{1}, \ldots, f_{s}\right)$ is a tuple of coherently $Z$-separating polynomials, where we have $c_{i}=\operatorname{LC}_{\sigma}\left(f_{i}\right)$ and $z_{i}=\operatorname{LT}_{\sigma}\left(f_{i}\right)$ for $i=1, \ldots, s$, and where $\left\{g_{1}, \ldots, g_{t}\right\}$ is the reduced $\hat{\sigma}$-Gröbner basis of $I \cap \widehat{P}$.

In polynomial system solving, the term ordering Lex frequently acts as a separating term ordering, as in the following example.

Example 2.10. Let $K$ be a perfect field, let $P=K\left[x_{1}, \ldots, x_{n}\right]$, and let $I \subseteq \mathfrak{M}$ be a 0 -dimensional radical ideal in normal $x_{n}$-position. Then the Shape Lemma (see [8], Thm. 3.7.25) says that $\sigma=$ Lex is a $Z$-separating term ordering for $I$ when $Z=\left\{x_{1}, \ldots, x_{n-1}\right\}$.

Notice that a $Z$-separating Gröbner basis consists of two parts, both of which are reduced Gröbner bases. However, the full set need not be the reduced $\sigma$-Gröbner basis of $I$, as the following example shows.

Example 2.11. Let $P=\mathbb{Q}[x, y, z]$, let $F=\left\{x-y^{2}\right\}$, let $I=\left\langle x-y^{2}, y^{2}-z^{3}\right\rangle$, and let $\sigma=$ Lex. Then $F$ is the reduced $\sigma$-Gröbner basis of $\langle F\rangle$ and $\left\{y^{2}-z^{3}\right\}$ is the reduced $\hat{\sigma}$-Gröbner basis of $I \cap \mathbb{Q}[y, z]$. Thus $\left\{x-y^{2}, y^{2}-z^{3}\right\}$ is a $Z$-separating Lex-Gröbner basis of $I$ for $Z=\{x\}$, but it is not equal to the reduced Lex-Gröbner basis $\left\{x-z^{3}, y^{2}-z^{3}\right\}$ of $I$.

The exact relation between a $Z$-separating Gröbner basis and the corresponding reduced Gröbner basis of an ideal is given by the following proposition.

Proposition 2.12. In the setting of Definition [2.9, let $\sigma$ be a Z-separating term ordering for an ideal $I \subset \mathfrak{M}$.

(a) The reduced $\sigma$-Gröbner basis of $I$ is a $Z$-separating $\sigma$-Gröbner basis of $I$.

(b) Let $\left\{\frac{1}{c_{1}} f_{1}, \ldots, \frac{1}{c_{s}} f_{s}, g_{1}, \ldots, g_{t}\right\}$ be a $Z$-separating $\sigma$-Gröbner basis of $I$. For $i=1, \ldots, s$, let $h_{i}=\mathrm{NF}_{\hat{\sigma}, I \cap \widehat{P}}\left(\right.$ tail $\left._{z_{i}}\left(f_{i}\right)\right)$. Then the reduced $\sigma$-Gröbner basis of $I$ is given by $H=\left\{z_{1}-h_{1}, \ldots, z_{s}-h_{s}, g_{1}, \ldots, g_{t}\right\}$.

Proof. First we show (a). Since $\sigma$ is a coherently $Z$-separating term ordering, we know that $\left\{z_{1}, \ldots, z_{s}\right\}$ is part of a minimal set of generators of $\operatorname{LT}_{\sigma}(I)$. Hence the reduced $\sigma$-Gröbner basis $G$ of $I$ is of the form $G=\left\{f_{1}, \ldots, f_{s}, g_{1}, \ldots, g_{t}\right\}$ with $\operatorname{LC}_{\sigma}\left(f_{i}\right)=1$ and $\operatorname{LT}_{\sigma}\left(f_{i}\right)=z_{i}$ for $i=1, \ldots, s$, and with $\operatorname{LT}_{\sigma}\left(g_{j}\right) \in \widehat{P}$ 
for $j=1, \ldots, t$. Since $G$ is fully interreduced, we have $z_{i} \notin \operatorname{indets}\left(\operatorname{tail}_{z_{i}}\left(f_{i}\right)\right)$ and $z_{i} \notin \operatorname{indets}\left(f_{j}\right)$ for $i=1, \ldots, s$ and $j \neq i$, as well as $z_{i} \notin \operatorname{indets}\left(g_{k}\right)$ for $k=1, \ldots, t$. Therefore $G$ is a $Z$-separated $\sigma$-Gröbner basis of $I$.

To prove (b), it suffices to show that $H$ is fully interreduced. Using $g_{j} \in \widehat{P}$ for $j=1, \ldots, t$, we know that $g_{j}$ cannot be reduced by any element in $F=$ $\left\{\frac{1}{c_{1}} f_{1}, \ldots, \frac{1}{c_{s}} f_{s}\right\}$. Furthermore, as $F$ is fully interreduced, it remains to reduce the elements of $F$ with respect to $\widehat{G}=\left\{g_{1}, \ldots, g_{t}\right\}$. This is achieved by replacing $\operatorname{tail}_{z_{i}}\left(f_{i}\right)$ by $\mathrm{NF}_{\hat{\sigma}, I \cap \widehat{P}}\left(\operatorname{tail}_{z_{i}}\left(f_{i}\right)\right)$ which is its normal remainder under division by $\widehat{G}$. Altogether, it follows that $H$ is the reduced $\sigma$-Gröbner basis of $I$.

Now we are ready to prove the main result of this section. It shows that a $Z$-separating Gröbner basis allows us to embed the affine scheme defined by a polynomial ideal into a lower dimensional affine space. Recall that, for a set of distinct indeterminates $Z=\left\{z_{1}, \ldots, z_{s}\right\}$ in $X$, Notation 2.4 provides a disjoint union $X=Y \cup Z$, and that we let $\widehat{P}=K[Y]=K\left[y_{1}, \ldots, y_{n-s}\right]$.

\section{Theorem 2.13. (The $Z$-Separating Re-embedding)}

Let $I \subseteq \mathfrak{M}$ be an ideal, let $Z=\left\{z_{1}, \ldots, z_{s}\right\}$ be a set of distinct indeterminates in $X$, let $\sigma$ be a $Z$-separating term ordering for $I$, and let $\left\{\frac{1}{c_{1}} f_{1}, \ldots, \frac{1}{c_{s}} f_{s}\right.$, $\left.g_{1}, \ldots, g_{t}\right\}$ be a $Z$-separating Gröbner basis of $I$, where $\left(f_{1}, \ldots, f_{s}\right)$ is a coherently $Z$-separated tuple of polynomials, where $c_{i}=\operatorname{LT}_{\sigma}\left(f_{i}\right)$ and $z_{i}=\operatorname{LT}_{\sigma}\left(f_{i}\right)$ for $i=1, \ldots, s$, and where $\left\{g_{1}, \ldots, g_{t}\right\}$ is the reduced $\hat{\sigma}$-Gröbner basis of $I \cap \widehat{P}$.

(a) Let $\varphi$ be the $K$-algebra homomorphism $\varphi: P \longrightarrow \widehat{P}$ given by $x_{i} \mapsto y_{j}$ if $y_{j}=x_{i}$ for some $j \in\{1, \ldots, n-s\}$ and by $x_{i} \mapsto \operatorname{tail}_{z_{j}}\left(f_{j}\right)$ if $z_{j}=x_{i}$ for some $j \in\{1, \ldots, s\}$. Then $\varphi$ induces an isomorphism of $K$-algebras

$$
\Phi: P / I \longrightarrow \widehat{P} /(I \cap \widehat{P})
$$

which is defined by $\Phi\left(x_{i}+I\right)=\varphi\left(x_{i}\right)+(I \cap \widehat{P})$ for $i=1, \ldots, n$.

(b) The map $\Phi^{-1}: \widehat{P} /(I \cap \widehat{P}) \longrightarrow P / I$ is given by $y_{i}+(I \cap \widehat{P}) \mapsto y_{i}+I$ for $i=1, \ldots, n-s$.

(c) The maps $\Phi$ and $\Phi^{-1}$ do not depend on the choice of a $Z$-separating Gröbner basis of $I$. In particular, we may use the reduced $\sigma$-Gröbner basis of $I$ to define them.

Proof. To prove (a), we consider the composition $\psi$ of $\varphi$ with the canonical epimorphism $\widehat{P} \longrightarrow \widehat{P} /(I \cap \widehat{P})$ and show that $I=\operatorname{Ker}(\psi)$. To prove the inclusion $\subseteq$, we note that $\frac{1}{c_{i}} f_{i}=z_{i}-\operatorname{tail}_{z_{i}}\left(f_{i}\right)$, and that $\operatorname{tail}_{z_{i}}\left(f_{i}\right) \in \widehat{P}$ implies

$$
\psi\left(\frac{1}{c_{i}} f_{i}\right)=\operatorname{tail}_{z_{i}}\left(f_{i}\right)-\operatorname{tail}_{z_{i}}\left(f_{i}\right)+(I \cap \widehat{P})=0
$$

for $i=1, \ldots, s$. Furthermore, since $g_{j} \in I \cap \widehat{P}$, we also have $\psi\left(g_{j}\right)=0$ for $j=1, \ldots, t$.

To show the inclusion $\supseteq$, we take a polynomial $h \in \operatorname{Ker}(\psi)$ and note that $\tilde{h}=\mathrm{NF}_{\sigma, I}(h) \in \widehat{P}$. Hence there exist $k_{1}, \ldots, k_{s}, \ell_{1}, \ldots, \ell_{t} \in P$ such that $h=$ $k_{1} f_{1}+\cdots+k_{s} f_{s}+\ell_{1} g_{1}+\cdots+\ell_{t} g_{t}+\tilde{h}$. Since we have shown already that $\psi\left(f_{i}\right)=$ $\psi\left(g_{j}\right)=0$, it follows that $\psi(\tilde{h})=0$, and hence $\tilde{h} \in I$. Altogether, we get $h \in I$, as claimed.

Using the equality $I=\operatorname{Ker}(\psi)$, we see that $\psi$ induces an injective $K$-algebra homomorphism $\Phi: P / I \longrightarrow \widehat{P} /(I \cap \widehat{P})$ which is also surjective, because $\psi\left(y_{i}\right)=$ $y_{i}+(I \cap \widehat{P})$ for $i=1, \ldots, n-s$.

Claim (b) follows immediately from (a). To show (c), it suffices to prove that the map $\Phi$ defined via the set $\left\{\frac{1}{c_{1}} f_{1}, \ldots, \frac{1}{c_{s}} f_{s}, g_{1}, \ldots, g_{t}\right\}$ agrees with the map $\Psi$ defined analogously via the reduced $\sigma$-Gröbner basis $G$ of $I$, because then an 
application of Proposition 2.12, a finishes the proof. By part (b) of this proposition, the set $G$ is of the form $G=\left\{f_{1}^{\prime}, \ldots, f_{s}^{\prime}, g_{1}, \ldots, g_{t}\right\}$ where $f_{i}^{\prime}=z_{i}-h_{i}$ and $h_{i}=\mathrm{NF}_{\hat{\sigma}, I \cap \widehat{P}}\left(\operatorname{tail}_{z_{i}}\left(f_{i}\right)\right)$ for $i=1, \ldots, s$. For an indeterminate $x_{i}$ such that $i \in\{1, \ldots, n\}$ and $x_{i}=z_{j}$ with $j \in\{1, \ldots, s\}$, it follows that

$$
\Phi\left(x_{i}\right)=\operatorname{tail}_{z_{j}}\left(f_{j}\right)+I \cap \widehat{P}=h_{j}+I \cap \widehat{P}=\Psi\left(x_{i}\right)
$$

because the polynomial tail $z_{j}\left(f_{j}\right)$ and its normal form with respect to $I \cap \widehat{P}$ differ by an element of $I \cap \widehat{P}$. Consequently, we get $\Phi=\Psi$, as claimed.

The isomorphism $\Phi$ constructed in part (a) of this theorem seems to depend on the fact that we fixed a $Z$-separating term ordering $\sigma$ for the given ideal $I$. Our next proposition shows that it is in fact independent of the choice of $\sigma$.

Proposition 2.14. Let $I \subseteq \mathfrak{M}$ be an ideal in $P$, let $Z=\left\{z_{1}, \ldots, z_{s}\right\}$ be a set of distinct indeterminates in $\mathbb{X}$, and let $\sigma, \sigma^{\prime}$ be $Z$-separating term orderings for $I$. Then the corresponding $K$-algebra isomorphisms $\Phi, \Phi^{\prime}: P / I \longrightarrow \widehat{P} /(I \cap \widehat{P})$ are equal.

Proof. According to Theorem 2.13. c, we may use the reduced Gröbner bases of $I$ with respect to $\sigma, \sigma^{\prime}$ to define $\Phi, \Phi^{\prime}$ and write them as $G=\left\{f_{1}, \ldots, f_{s}, g_{1}, \ldots, g_{t}\right\}$ and $G^{\prime}=\left\{f_{1}^{\prime}, \ldots, f_{s}^{\prime}, g_{1}^{\prime}, \ldots, g_{t^{\prime}}^{\prime}\right\}$, where $\left(f_{1}, \ldots, f_{s}\right)$ and $\left(f_{1}^{\prime}, \ldots, f_{s}^{\prime}\right)$ are coherently $Z$-separated and where $\left\{g_{1}, \ldots, g_{t}\right\}$ and $\left\{g_{1}^{\prime}, \ldots, g_{t^{\prime}}^{\prime}\right\}$ are the reduced Gröbner bases of $I \cap \widehat{P}$ with respect to $\sigma$ and $\sigma^{\prime}$.

For every $x_{i} \in X \backslash Z$, we have $\Phi\left(x_{i}+I\right)=x_{i}+(I \cap \widehat{P})=\Phi^{\prime}\left(x_{i}+I\right)$. Now we consider $z_{i} \in Z$. We have $\Phi\left(z_{i}+I\right)=\operatorname{tail}_{z_{i}}\left(f_{i}\right)+(I \cap \widehat{P})$ and $\Phi^{\prime}\left(z_{i}+I\right)=$ $\operatorname{tail}_{z_{i}}\left(f_{i}^{\prime}\right)+(I \cap \widehat{P})$. Since $\operatorname{tail}_{z_{i}}\left(f_{i}\right)-\operatorname{tail}_{z_{i}}\left(f_{i}^{\prime}\right)=f_{i}-f_{i}^{\prime} \in I \cap \widehat{P}$, we obtain $\Phi\left(z_{i}+I\right)=\Phi^{\prime}\left(z_{i}+I\right)$, and the proof is complete.

This proposition motivates the following definition.

Definition 2.15. Let $I \subseteq \mathfrak{M}$ be an ideal, let $Z=\left\{z_{1}, \ldots, z_{s}\right\}$ be a set of distinct indeterminates in $X$, and let $\varepsilon: \widehat{P} \longrightarrow \widehat{P} /(I \cap \widehat{P})$ be the canonical epimorphism.

(a) The $K$-algebra isomorphism $\Phi: P / I \longrightarrow \widehat{P} /(I \cap \widehat{P})$ defined in Theorem 2.13, a is called the $Z$-separating re-embedding of $I$.

(b) The map $\operatorname{Spec}\left(\Phi^{-1} \circ \varepsilon\right): \mathbb{X} \longrightarrow \mathbb{A}_{K}^{n-s}$ is called the $Z$-separating reembedding of $\mathbb{X}$.

By Proposition 2.14 the $Z$-separating re-embedding of $I$ (or of $\mathbb{X}$ ) does not depend on the choice of a $Z$-separating term ordering $\sigma$. This is why we did not mention the choice of $\sigma$ in this definition. Clearly, the $Z$-separating re-embedding of $\mathbb{X}$ is obtained from the given embedding $\mathbb{X} \subseteq \mathbb{A}_{K}^{n}$ via the projection along the linear subspace defined by $Z$. The following example illustrates the theorem.

Example 2.16. In the setting of Example 2.8, the reduced $\sigma$-Gröbner basis of $I=\left\langle f_{1}, f_{2}\right\rangle$ is $G=\left\{-f_{1}, g\right\}$. Here we have $Z=\{y, z\}$ and $\widehat{P}=\mathbb{Q}[x]$ as well as $I \cap \widehat{P}=\langle 0\rangle$. Hence the $Z$-separating re-embedding of $I$ is the map $\Phi: P / I \longrightarrow$ $\mathbb{Q}[x]$ defined by $x+I \mapsto x$, by $y+I \mapsto \operatorname{tail}_{y}\left(-f_{1}\right)$, and by $z+I \mapsto \operatorname{tail}_{z}(g)$. Moreover, the $Z$-separating re-embedding of $\mathbb{X}=\operatorname{Spec}(P / I)$ yields an isomorphism $\mathbb{X} \cong \mathbb{A}_{K}^{1}$. 


\section{Separating Re-embeddings and the Gröbner Fan}

In this section we consider the problem of finding good embeddings of affine schemes into affine spaces. As before, we let $K$ be a field, let $P=K\left[x_{1}, \ldots, x_{n}\right]$, let $\mathfrak{M}=\left\langle x_{1}, \ldots, x_{n}\right\rangle$, let $I$ be an ideal in $P$ which is contained in $\mathfrak{M}$, let $R=P / I$, and let $\mathbb{X}=\operatorname{Spec}(R)$. The epimorphism $P \longrightarrow P / I$ corresponds to an embedding of $\mathbb{X}$ into the affine space $\mathbb{A}_{K}^{n}$. In this setting we call $n=\operatorname{dim}(P)$ the ambient dimension of the embedding of $\mathbb{X}$ or of the presentation $P / I$.

Our goal can then be restated by saying that we look for isomorphisms of affine $K$-algebras $\varphi: P / I \cong P^{\prime} / I^{\prime}$ such that $P^{\prime}=K\left[y_{1}, \ldots, y_{m}\right]$, such that $I^{\prime}$ is an ideal in $P^{\prime}$, and such that the ambient dimension $\operatorname{dim}\left(P^{\prime}\right)$ is as small as possible.

Example 3.1. In the setting of Example 2.3, we have $P=\mathbb{Q}[x, y, z]$ and $\operatorname{dim}(P)=$ 3. We constructed an isomorphism $P / I \cong P^{\prime} / I^{\prime}$, where we have $P^{\prime}=\mathbb{Q}[y, z]$ and $I^{\prime}=\left\langle y^{4} z-y z^{4}+2 z^{2}-2 y\right\rangle$. Since $\operatorname{dim}\left(P^{\prime}\right)=2$, we have re-embedded $\mathbb{X}=\operatorname{Spec}(P / I)$ into an affine space with a lower dimension.

Example 3.2. In the setting of Examples 2.8 and 2.16, we have re-embedded the scheme $\mathbb{X}=\operatorname{Spec}\left(\mathbb{Q}[x, y, z] /\left\langle x^{2}-x-y, y^{2}-z\right\rangle\right)$ from ambient dimension 3 to ambient dimension 1 . Since we actually found that $\mathbb{X} \cong \mathbb{A}_{K}^{1}$, the ambient dimension of the new embedding is as small as possible.

In view of our goal to find presentations of $R=P / I$ with minimal ambient dimensions, we introduce the following terminology.

Definition 3.3. Let $R=P / I$ be an affine $K$-algebra as above.

(a) A $K$-algebra isomophism $\Psi: P / I \longrightarrow P^{\prime} / I^{\prime}$, where $P^{\prime}$ is a polynomial ring over $K$ and $I^{\prime}$ is an ideal in $P^{\prime}$, is called a re-embedding of $I$.

(b) A re-embedding $\Psi: P / I \longrightarrow P^{\prime} / I^{\prime}$ of $I$ is called an optimal reembedding if every $K$-algebra isomorphism $P / I \longrightarrow P^{\prime \prime} / I^{\prime \prime}$ with a polynomial ring $P^{\prime \prime}$ over $K$ and an ideal $I^{\prime \prime}$ in $P^{\prime \prime}$ satisfies $\operatorname{dim}\left(P^{\prime \prime}\right) \geq \operatorname{dim}\left(P^{\prime}\right)$.

(c) For an optimal re-embedding $\Psi: P / I \longrightarrow P^{\prime} / I^{\prime}$ of $I$, we call $\operatorname{edim}(R)=$ $\operatorname{dim}\left(P^{\prime}\right)$ the embedding dimension of the affine $K$-algebra $R$.

(d) A $Z$-separating re-embedding $\Phi: P / I \longrightarrow \widehat{P} /(I \cap \widehat{P})$ is called an optimal separating re-embedding, if for every subset $Z^{\prime} \subseteq X$ such that $I$ contains a tuple of $Z^{\prime}$-separating polynomials, we have $\# Z^{\prime} \leq \# Z$.

(e) For an optimal $Z$-separating re-embedding $\Phi: P / I \longrightarrow \widehat{P} /(I \cap \widehat{P})$, we call $\operatorname{sepdim}(P / I)=\operatorname{dim}(\widehat{P})=n-\# Z$ the separating embedding dimension of the presentation $P / I$ of $R$.

At this point it is clear that, to find optimal separating re-embedding of $I$, we need to find term orderings having the maximum number of elements in their reduced Gröbner bases of $I$ whose leading terms are indeterminates. To find such reduced Gröbner bases we use the notion of the Gröbner fan of the ideal $I$ which was introduced in [13. Formally, it is a subdivision of the closed non-negative orthant $\mathbb{R}_{+}^{n}$ consisting of a finite number of polyhedral cones, such that the cones are in one-to-one correspondence with the leading term ideals of $I$. We can also associate to each polyhedral cone a marked reduced Gröbner basis of $I$ and use these Gröbner bases to describe the Gröbner fan in the following way. The notion of marked reduced Gröbner basis was introduced in [15].

Definition 3.4. Let $I$ be a proper ideal in $P=K\left[x_{1}, \ldots, x_{n}\right]$.

(a) Given a term ordering $\sigma$, a marked $\sigma$-Gröbner basis of $I$ is a set of pairs $\bar{G}=\left\{\left(\operatorname{LT}_{\sigma}\left(g_{1}\right), g_{1}\right), \ldots,\left(\operatorname{LT}_{\sigma}\left(g_{r}\right), g_{r}\right)\right\}$, where $G=\left\{g_{1}, \ldots, g_{r}\right\}$ is a $\sigma$-Gröbner basis of $I$. 
(b) Given a term ordering $\sigma$ and the marked reduced $\sigma$-Gröbner basis $\bar{G}=$ $\left\{\left(\operatorname{LT}_{\sigma}\left(g_{1}\right), g_{1}\right), \ldots,\left(\operatorname{LT}_{\sigma}\left(g_{r}\right), g_{r}\right)\right\}$ of $I$, we call

$$
\operatorname{LI}(\bar{G})=\left\{z \in X \mid \operatorname{LT}_{\sigma}\left(g_{i}\right)=z \quad \text { for some } i \in\{1, \ldots, r\}\right\}
$$

the set of leading indeterminates of $\bar{G}$.

(c) The Gröbner fan of $I$ is the set of all distinct marked reduced Gröbner bases of $I$. We denote it by $\operatorname{GFan}(I)$.

The following example shows that it is important to distinguish between reduced Gröbner bases and marked reduced Gröbner bases of $I$.

Example 3.5. In the $\operatorname{ring} P=K[x, y]$, consider the ideal $I=\langle x+y\rangle$. Then $I$ has only one reduced Gröbner basis, namely $\{x+y\}$, but two marked reduced Gröbner bases, namely $\{(x, x+y)\}$ and $\{(y, x+y)\}$. Hence the Gröbner fan of $I$ consists of two distinct elements in this case.

Using the language of Gröbner fans, we can rephrase some of the preceding considerations as follows.

Proposition 3.6. (Gröbner Fans and Optimal Separating Re-embeddings) Let $I \subseteq \mathfrak{M}$ be an ideal in $P$, and let $\operatorname{GFan}(I)=\left\{\bar{G}_{1}, \ldots, \bar{G}_{k}\right\}$.

(a) For $i=1, \ldots, k$, we have $\operatorname{sepdim}(P / I) \leq n-\# \operatorname{LI}\left(\bar{G}_{i}\right)$.

(b) For $i \in\{1, \ldots, k\}$, we have $\operatorname{sepdim}(P / I)=n-\# \operatorname{LI}\left(\bar{G}_{i}\right)$ if and only if $\# \operatorname{LI}\left(\bar{G}_{i}\right)$ is maximal.

(c) For $i \in\{1, \ldots, k\}$ such that $\# \mathrm{LI}\left(\bar{G}_{i}\right)$ is maximal, the $\operatorname{LI}(\bar{G})$-separating re-embedding $\Phi: P / I \longrightarrow \widehat{P} /(I \cap \widehat{P})$ is optimal.

Proof. This follows immediately from Theorem 2.13

The following example shows that an optimal separating re-embedding need not be an optimal re-embedding of $I$.

Example 3.7. Let $P=\mathbb{Q}[x, y, z]$, and let $I=\left\langle f_{1}, f_{2}\right\rangle$, where

$$
\begin{aligned}
f_{1}= & x^{2}-2 x y-y^{2}+x-2 z \\
f_{2}= & x^{3} y+\frac{7}{6} x^{2} y^{2}+\frac{2}{3} x y^{3}+\frac{1}{6} y^{4}-\frac{1}{6} x^{3}+\frac{1}{3} x^{2} y+\frac{2}{3} x y^{2}+\frac{1}{3} y^{3}+\frac{1}{3} x^{2} z-\frac{1}{3} x y \\
& -\frac{1}{6} y-\frac{1}{6} z
\end{aligned}
$$

Then a computation using CoCoA shows that $\operatorname{GFan}(I)$ consists of 26 different marked reduced Gröbner bases. Only two of them define optimal separating reembeddings $\Phi: P / I \longrightarrow \widehat{P} /(I \cap \widehat{P})$.

One of these two bases is $\bar{G}=\left\{\left(z, g_{1}\right),\left(x^{4}, g_{2}\right)\right\}$ where

$$
\begin{aligned}
g_{1}= & z-\frac{1}{2} x^{2}+x y+\frac{1}{2} y^{2}-\frac{1}{2} x, \\
g_{2}= & x^{4}+4 x^{3} y+6 x^{2} y^{2}+4 x y^{3}+y^{4}+2 x^{2} y+4 x y^{2}+2 y^{3}-\frac{1}{2} x^{2}-x y+\frac{1}{2} y^{2} \\
& -\frac{1}{2} x-y
\end{aligned}
$$

Thus we get an optimal separating re-embedding $\Phi: P / I \longrightarrow \widehat{P} /(I \cap \widehat{P})$, where $\widehat{P} /(I \cap \widehat{P})$ has ambient dimension 2 .

Next we consider the $\mathbb{Q}$-algebra automorphism $\Psi: P \longrightarrow P$ given by $\Psi(x)=$ $x-y+x^{2}, \Psi(y)=y-x^{2}$, and $\Psi(z)=z+x^{4}+2 x^{3}-2 x^{2} y+x^{2}-2 x y+y^{2}$. Notice that its inverse $\Psi^{-1}: P \longrightarrow P$ is given by $\Psi^{-1}(x)=x+y, \Psi^{-1}(y)=y+(x+y)^{2}$, and $\Psi^{-1}(z)=z-x^{2}$. For $h_{1}=\Psi\left(g_{1}\right)$ and $h_{2}=\Psi\left(g_{2}\right)$, we have

$$
\begin{aligned}
h_{1}= & x-y-2 z \\
h_{2}= & -\frac{1}{6} x^{5}+\frac{1}{6} x^{4} y+\frac{1}{3} x^{4} z-\frac{1}{3} x^{4}+\frac{2}{3} x^{3} y-\frac{1}{3} x^{2} y^{2}+\frac{2}{3} x^{3} z-\frac{2}{3} x^{2} y z-\frac{1}{6} x^{3} \\
& +\frac{1}{2} x^{2} y-\frac{1}{2} x y^{2}+\frac{1}{6} y^{3}+\frac{1}{3} x^{2} z-\frac{2}{3} x y z+\frac{1}{3} y^{2} z+\frac{1}{6} y^{2}+\frac{1}{6} y-\frac{1}{6} z
\end{aligned}
$$

Consequently, the map $\Psi$ induces a $K$-algebra isomorphism $\psi: P / I \longrightarrow P / I^{\prime}$, where $I^{\prime}=\left\langle h_{1}, h_{2}\right\rangle$. 
Next we calculate that GFan $\left(I^{\prime}\right)$ comprises five marked reduced Gröbner bases. The optimal separating re-embedding $\Phi^{\prime}: P / I^{\prime} \longrightarrow \widehat{P}^{\prime} /\left(I^{\prime} \cap \widehat{P}^{\prime}\right)$ of $I^{\prime}$ is obtained using the Gröbner basis $\left\{z-y^{2}+y, x-2 y^{2}+y\right\}$. It defines a separating reembedding $\Phi^{\prime}: P / I^{\prime} \longrightarrow \mathbb{Q}[y]$.

In summary, we have a $\mathbb{Q}$-algebra isomorphism $\Phi^{\prime} \circ \psi: P / I \longrightarrow \mathbb{Q}[y]$ and conclude that $\operatorname{edim}(P / I)=1$, whereas $\operatorname{sepdim}(P / I)=3-\# \operatorname{LI}(\bar{G})=2$.

Several marked reduced Gröbner bases can correspond to the same separating reembedding, as Proposition 2.14 indicates. Hence it is natural to define the following equivalence relation.

Definition 3.8. Let $I \subseteq \mathfrak{M}$ be an ideal in $P$, and let $\operatorname{GFan}(I)=\left\{\bar{G}_{1}, \ldots, \bar{G}_{k}\right\}$. Then the equivalence relation $\sim_{\text {LI }}$ on $\operatorname{GFan}(I)$ defined by

$$
\bar{G}_{i} \sim_{\mathrm{LI}} \bar{G}_{j} \Longleftrightarrow \operatorname{LI}\left(\bar{G}_{i}\right)=\operatorname{LI}\left(\bar{G}_{j}\right)
$$

is called the leading indeterminate set equivalence on $\operatorname{GFan}(I)$.

The leading indeterminate set equivalence allows us to classify separating reembeddings and optimal separating re-embeddings as follows.

Proposition 3.9. Let $I \subseteq \mathfrak{M}$ be an ideal in $P$.

(a) There is a 1-1 correspondence between $Z$-separating re-embeddings $\Phi: P / I \longrightarrow \widehat{P} /(I \cap \widehat{P})$ of $I$ and the set of equivalence classes $\operatorname{GFan}(I) / \sim_{\mathrm{LI}}$.

(b) The equivalence relation $\sim_{\mathrm{LI}}$ induces an equivalence relation on the set $\mathcal{G}$ of all $\bar{G} \in \operatorname{GFan}(I)$ such that $\operatorname{sepdim}(P / I)=n-\# \operatorname{LI}(\bar{G})$.

(c) There is a 1-1 correspondence between optimal separating re-embeddings $\Phi: P / I \longrightarrow \widehat{P} /(I \cap \widehat{P})$ of $I$ and the set of equivalence classes $\mathcal{G} / \sim_{\mathrm{LI}}$.

Proof. To prove (a), we note that Proposition 2.14 says that equivalent marked reduced Gröbner bases of $I$ yield identical maps $\Phi$. Conversely, if we are given two such re-embeddings to the same ring $\widehat{P} /(I \cap \widehat{P})$, where $\widehat{P}=K[Y]$, then they correspond to the same set of indeterminates $Z=X \backslash Y$ and hence to equivalent marked reduced Gröbner bases. Claims (b) and (c) follow immediately from (a).

The next example illustrates this proposition.

Example 3.10. Let $P=\mathbb{Q}[x, y, z]$, and let $I=\left\langle f_{1}, f_{2}, f_{3}, f_{4}, f_{5}, f_{6}\right\rangle$, where we have $f_{1}=x^{2}-y, f_{2}=x y-x-z, f_{3}=y^{2}+z^{2}+2 x+y+2 z, f_{4}=x z+z^{2}+2 x+2 y+2 z$, $f_{5}=y z+z^{2}+3 x+3 y+3 z$, and $f_{6}=z^{3}+z^{2}-5 x-5 y-5 z$.

Using CoCoA, we may check that GFan $(I)$ consists of 13 marked reduced Gröbner bases and is the disjoint union of four equivalence classes modulo $\sim_{\text {LI }}$. These equivalence classes are represented by the following marked reduced Gröbner bases:

$$
\begin{aligned}
& \bar{G}_{1}=\left\{\left(x, x+\frac{1}{3} y z+\frac{1}{3} z^{2}+y+z\right),\left(y^{2}, y^{2}-\frac{2}{3} y z+\frac{1}{3} z^{2}-y\right),\right. \\
&\left.\left(y z^{2}, y z^{2}+\frac{10}{3} y z-\frac{2}{3} z^{2}\right),\left(z^{3}, z^{3}+\frac{5}{3} y z+\frac{8}{3} z^{2}\right)\right\} \\
& \bar{G}_{2}=\left\{(z, z-x y+x),\left(x^{2}, x^{2}-y\right),\left(y^{3}, y^{3}+2 x y-y^{2}+2 y\right),\right.\left.\left(x y^{2}, x y^{2}-y^{2}+2 y\right)\right\} \\
& \bar{G}_{3}=\left\{\left(y, y+\frac{1}{2} x z+\frac{1}{2} z^{2}+x+z\right),\left(x^{2}, x^{2}+\frac{1}{2} x z+\frac{1}{2} z^{2}+x+z\right),\right. \\
& \\
&\left.\left(x z^{2}, x z^{2}+\frac{5}{2} x z-\frac{1}{2} z^{2}\right),\left(z^{3}, z^{3}+\frac{5}{2} x z+\frac{7}{2} z^{2}\right)\right\} \\
& \bar{G}_{4}=\left\{\left(y, y-x^{2}\right),\left(z, z-x^{3}+x\right),\left(x^{5}, x^{5}-x^{4}+2 x^{2}\right)\right\}
\end{aligned}
$$

Here the equivalence class of $\bar{G}_{4}$ contains only one marked reduced Gröbner basis, namely $\bar{G}_{4}$. It is the only equivalence class which defines an optimal separating re-embedding of $I$. Here we have $Z=\{y, z\}$ and the resulting separating reembedding is the map $\Phi: P / I \longrightarrow \mathbb{Q}[x] / I^{\prime}$ given by $x+I \mapsto x+I^{\prime}, y+I \mapsto x^{2}+I^{\prime}$ 
and $z+I \mapsto x^{3}-x+I^{\prime}$, where $I^{\prime}=\left\langle x^{5}-x^{4}+2 x^{2}\right\rangle$. In particular, we see that $\# \operatorname{LI}\left(\bar{G}_{4}\right)=2$, and hence $\operatorname{sepdim}(P / I)=1$.

Notice that in the current example we even have $\operatorname{edim}(P / I)=1$, since the ring $P / I \cong \mathbb{Q}[x] / I^{\prime}$ has a singularity at the origin (see also Example 4.4).

\section{Cotangent Spaces and Optimal Re-embeddings}

In this section we investigate criteria for checking whether an optimal separating re-embedding yields already an optimal re-embedding. As we saw in Example 3.7. this is not always the case. However, we can use the size of the cotangent space at a linear maximal ideal to bound the embedding dimension and the separating embedding dimension, as the next theorem shows.

In the following we continue to use the setting introduced above. In particular, let $K$ be a field, let $P=K\left[x_{1}, \ldots, x_{n}\right]$, let $I$ be an ideal in $P$ contained in $\mathfrak{M}=\left\langle x_{1}, \ldots, x_{n}\right\rangle$, let $R=P / I$, and let $\mathfrak{m}=\mathfrak{M} / I$.

Theorem 4.1. In the above setting, let $\bar{G}$ be a marked reduced Gröbner basis of I.

(a) We have $\# \operatorname{LI}(\bar{G}) \leq \operatorname{dim}_{K}\left(\operatorname{Lin}_{\mathfrak{M}}(I)\right)$.

(b) We have $\operatorname{dim}_{K}\left(\operatorname{Cot}_{\mathfrak{m}}(P / I)\right) \leq \operatorname{edim}(P / I) \leq \operatorname{sepdim}(P / I) \leq n-\# \operatorname{LI}(\bar{G})$.

Proof. To prove (a), we let $\bar{G}$ be a $Z$-separating Gröbner basis of $I$ such that $\# \operatorname{LI}(\bar{G})=\# Z$ is maximal. It follows that the non-zero $\mathfrak{M}$-linear parts $\operatorname{Lin}_{\mathfrak{M}}(g)$ of the elements $g \in \bar{G}$ have the elements of $Z$ as their leading terms. Hence they are $K$-linearly independent, and Proposition 1.6 implies that they are contained in a $K$-basis of $\operatorname{Lin}_{\mathfrak{M}}(I)$. This yields the claim.

To show (b), we note that the second and third inequalities follow from the definitions and Theorem 2.13. To prove the first inequality, we note that both $\operatorname{edim}(R)$ and the cotangent space $\operatorname{Cot}_{\mathfrak{m}}(R)=\mathfrak{m} / \mathfrak{m}^{2}$ are invariants of the ring $R=$ $P / I$ and do not depend on the particular presentation. Hence we may assume that $R=P / I$ is a presentation such that $\operatorname{dim}(P)=\operatorname{edim}(R)$. Then Proposition 1.5 yields $\operatorname{dim}_{K}\left(\operatorname{Cot}_{\mathfrak{m}}(P / I)\right)=n-\operatorname{dim}_{K}\left(\operatorname{Lin}_{\mathfrak{M}}(I)\right) \leq \operatorname{dim}(P)=\operatorname{edim}(R)$, as was to be shown.

A particularly powerful way to use this theorem is given by the following corollary.

Corollary 4.2. In the above setting, assume that $Z$ is a set of $s$ distinct indeterminates in $X$ and that there exists a coherently $Z$-separating tuple $\left(f_{1}, \ldots, f_{s}\right)$ of polynomials in I. If $s=\operatorname{dim}_{K}\left(\operatorname{Lin}_{\mathfrak{M}}(I)\right)$ then we have $\operatorname{edim}(P / I)=n-s$ and the $Z$-separating re-embedding $\Phi: P / I \longrightarrow \widehat{P} /(I \cap \widehat{P})$ is an optimal re-embedding.

Proof. The hypothesis implies $\operatorname{dim}_{K}\left(\operatorname{Cot}_{\mathfrak{m}}(P / I)=n-\operatorname{dim}_{K}\left(\operatorname{Lin}_{\mathfrak{M}}(I)\right)=n-\right.$ $s=n-\# \operatorname{LI}(\bar{G})$. Hence we have equalities in part (b) of the theorem, and thus $\operatorname{edim}(P / I)=n-s$.

The following examples provide some explicit instances for the application of this corollary.

Example 4.3. Let $P=\mathbb{Q}[x, y, z, w, t]$, let $I=\left\langle f_{1}, f_{2}, f_{3}, f_{4}\right\rangle$, where $f_{1}=x^{2}+$ $x-z+t, f_{2}=z^{2}-w^{2}-w, f_{3}=w^{2}-y+w-t$, and $f_{4}=x^{2}+w^{2}-w$, and let $\mathfrak{m}=\langle x, y, z, w, t\rangle / I$.

Using CoCoA, we may check that the Gröbner fan of $I$ comprises 462 marked reduced Gröbner bases. The number of leading indeterminate set equivalence classes is 7 , and the number of equivalence classes which correspond to optimal separating 
re-embeddings of $I$ is two. A marked reduced Gröbner basis which represents one of these two equivalence classes is

$$
\begin{aligned}
\bar{G}=\{ & \left(t, t+x^{2}+x-z\right),\left(y, y-x^{2}-z^{2}-x+z\right),\left(w, w-\frac{1}{2} x^{2}-\frac{1}{2} z^{2}\right), \\
& \left.\left(x^{4}, x^{4}+2 x^{2} z^{2}+z^{4}+2 x^{2}-2 z^{2}\right)\right\}
\end{aligned}
$$

Here we have $\# \operatorname{LI}(\bar{G})=3$ and $\operatorname{dim}(P)-\# \operatorname{LI}(\bar{G})=2$.

On the other hand, it is easy to check that $\operatorname{Lin}_{\mathfrak{M}}(I)=\langle x-z+t, y+t, w\rangle_{K}$. Therefore the corollary shows that $\operatorname{edim}(R)=2$ and the $Z$-separating re-embedding $\Phi: P / I \longrightarrow \mathbb{Q}[x, z] /\left\langle x^{4}+2 x^{2} z^{2}+z^{4}+2 x^{2}-2 z^{2}\right\rangle$ is an optimal re-embedding.

A similar argument works in the setting of Example 3.10 .

Example 4.4. As in Example [3.10 let $P=\mathbb{Q}[x, y, z]$, and let $I=\left\langle f_{1}, \ldots, f_{6}\right\rangle$, where we have $f_{1}=x^{2}-y, f_{2}=x y-x-z, f_{3}=y^{2}+z^{2}+2 x+y+2 z$, $f_{4}=x z+z^{2}+2 x+2 y+2 z, f_{5}=y z+z^{2}+3 x+3 y+3 z$, and $f_{6}=z^{3}+z^{2}-5 x-5 y-5 z$. We have already seen that, for $Z=\{y, z\}$, there is a $Z$-separating re-embedding $\Phi: P / I \longrightarrow \mathbb{Q}[x] /\left\langle x^{5}-x^{4}+2 x^{2}\right\rangle$.

Since $\operatorname{Lin}_{\mathfrak{M}}(I)=\langle x+z, y\rangle_{K}$, we get $\operatorname{dim}_{\mathbb{Q}}\left(\operatorname{Lin}_{\mathfrak{M}}(I)\right)=2$. Using the corollary, we conclude that $\operatorname{edim}(P / I)=1$ and that $\Phi$ is an optimal re-embedding.

Let us also note that any $Z$-separating re-embedding $\Phi: P / I \longrightarrow \widehat{P} /(I \cap \widehat{P})$ such that $I \cap \widehat{P}=\langle 0\rangle$ is an optimal re-embedding, since the cotangent space of the zero ideal in $\widehat{P}$ has dimension $\operatorname{dim}(\widehat{P})$. The next example shows that, in general, the problem of detecting an optimal embedding can be difficult to deal with.

Example 4.5. Let $P=\mathbb{Q}[x, y, z, t]$, and let $I=\langle f\rangle$, where $f=x+x^{2} y+z^{2}+t^{3}$. Clearly, the only reduced Gröbner basis of $I$ is $\{f\}$. As $f$ is not separating for any indeterminate, the optimal separating re-embedding of $P / I$ is the identity map.

But is it an optimal re-embedding, i.e., is $\operatorname{edim}(P / I)=4$ ? The answer is yes, but to prove this is not an easy task, as shown in 3 .

The final example contains an application of Corollary 4.2 to get an optimal re-embedding of a singular border basis scheme. In this example we use a bit of theory of border bases schemes (see for instance [9], [10, [11], [12], and [16]). As said in the introduction, we will devote another paper to an extensive study of good, possibly optimal, embeddings of border bases schemes.

Example 4.6. Let $P=\mathbb{Q}[x, y, z]$, and let $\mathcal{O}=\{1, z, y, x\}$. Then the border of $\mathcal{O}$ is given by $\partial \mathcal{O}=\left\{z^{2}, y z, x z, y^{2}, x y, x^{2}\right\}$. This yields $\mu=4$ and $\nu=6$. Consequently, the polynomial ring $\mathbb{Q}[C]$ has 24 indeterminates.

The computation of the linear part of $I\left(\mathbb{B}_{\mathcal{O}}\right)$ using Proposition 1.5 shows that we have $\operatorname{Lin}_{\mathfrak{M}}\left(I\left(\mathbb{B}_{\mathcal{O}}\right)\right)=\left\langle c_{11}, \ldots, c_{16}\right\rangle_{\mathbb{Q}}$, and therefore $n \mu=12$ implies that the monomial point $p=(0, \ldots, 0)$ is a singular point of $\mathbb{B}_{\mathcal{O}}$ (see also [12, Example 4.3). In particular, it follows that $\mathbb{B}_{\mathcal{O}}$ is not isomorphic to an affine space. Nevertheless, let us try to embed $\mathbb{B}_{\mathcal{O}}$ optimally. There exists a set of coherently $Z$-separating polynomials $\left\{f_{1}, \ldots, f_{6}\right\}$ in $I\left(\mathbb{B}_{\mathcal{O}}\right)$, where $Z=\left\{c_{11}, \ldots, c_{16}\right\}$ and

$$
\begin{aligned}
& f_{1}=c_{11}-c_{23} c_{41}-c_{33} c_{42}+c_{21} c_{43}-c_{43}^{2}+c_{31} c_{45}+c_{41} c_{46}, \\
& f_{2}=c_{12}-c_{23} c_{42}+c_{22} c_{43}-c_{33} c_{44}+c_{32} c_{45}-c_{43} c_{45}+c_{42} c_{46}, \\
& f_{3}=c_{13}-c_{26} c_{41}-c_{36} c_{42}+c_{23} c_{43}+c_{33} c_{45}, \\
& f_{4}=c_{14}-c_{25} c_{42}+c_{24} c_{43}-c_{35} c_{44}+c_{34} c_{45}-c_{45}^{2}+c_{44} c_{46}, \\
& f_{5}=c_{15}-c_{26} c_{42}+c_{25} c_{43}-c_{36} c_{44}+c_{35} c_{45}, \\
& f_{6}=c_{16}+c_{26} c_{32}-c_{25} c_{33}-c_{35}^{2}+c_{34} c_{36}-c_{36} c_{45}+c_{35} c_{46}
\end{aligned}
$$


Consequently, the $Z$-separating re-embedding of the ideal $I\left(\mathbb{B}_{\mathcal{O}}\right)$ is an isomorphism $\Phi: B_{\mathcal{O}} \rightarrow \widehat{P} /\left(I\left(\mathbb{B}_{\mathcal{O}}\right) \cap \widehat{P}\right)$, where $\widehat{P}=\mathbb{Q}\left[c_{21}, \ldots, c_{26}, c_{31}, \ldots, c_{36}, c_{41}, \ldots, c_{46}\right]$ is a polynomial ring in 18 indeterminates. Here it turns out that $I\left(\mathbb{B}_{\mathcal{O}}\right) \cap \widehat{P}$ is minimally generated by the 15 quadratic polynomials

$$
\begin{aligned}
& h_{1}=c_{25} c_{32}-c_{24} c_{33}+c_{26} c_{42}-c_{25} c_{43} \\
& h_{2}=c_{25} c_{41}-c_{23} c_{42}+c_{35} c_{42}-c_{33} c_{44} \\
& h_{3}=c_{25} c_{31}-c_{22} c_{33}-c_{36} c_{42}+c_{33} c_{45} \\
& h_{4}=c_{26} c_{31}-c_{23} c_{33}-c_{33} c_{35}+c_{32} c_{36}-c_{36} c_{43}+c_{33} c_{46} \\
& h_{5}=c_{22} c_{41}-c_{21} c_{42}+c_{32} c_{42}+c_{42} c_{43}-c_{31} c_{44}-c_{41} c_{45} \\
& h_{6}=c_{23} c_{24}-c_{22} c_{25}+c_{25} c_{34}-c_{24} c_{35}+c_{26} c_{44}-c_{25} c_{45} \\
& h_{7}=c_{23} c_{31}-c_{21} c_{33}+c_{32} c_{33}-c_{31} c_{35}-c_{36} c_{41}+c_{33} c_{43} \\
& h_{8}=c_{24} c_{41}-c_{22} c_{42}+c_{34} c_{42}-c_{32} c_{44}+c_{43} c_{44}-c_{42} c_{45} \\
& h_{9}=c_{23} c_{25}-c_{22} c_{26}+c_{25} c_{35}-c_{24} c_{36}+c_{26} c_{45}-c_{25} c_{46} \\
& h_{10}=c_{23} c_{32}-c_{22} c_{33}+c_{33} c_{34}-c_{32} c_{35}+c_{26} c_{41}-c_{23} c_{43}+c_{35} c_{43}-c_{33} c_{45} \\
& h_{11}=c_{24} c_{31}-c_{22} c_{32}-c_{23} c_{42}-c_{35} c_{42}+c_{22} c_{43}+c_{32} c_{45}-c_{43} c_{45}+c_{42} c_{46} \\
& h_{12}=c_{22} c_{23}-c_{21} c_{25}+c_{24} c_{33}-c_{22} c_{35}-c_{26} c_{42}+2 c_{25} c_{43}-c_{36} c_{44}-c_{23} c_{45}+c_{35} c_{45} \\
& h_{13}=c_{22}^{2}-c_{21} c_{24}+c_{24} c_{32}-c_{22} c_{34}+c_{24} c_{43}-c_{23} c_{44}-c_{35} c_{44}+c_{34} c_{45}-c_{45}^{2}+c_{44} c_{46} \\
& h_{14}=c_{22} c_{31}-c_{21} c_{32}+c_{32}^{2}-c_{31} c_{34}-c_{23} c_{41}-c_{35} c_{41}+c_{21} c_{43}-c_{43}^{2}+c_{31} c_{45}+c_{41} c_{46} \\
& h_{15}=c_{23}^{2}-c_{21} c_{26}+c_{26} c_{32}-c_{35}^{2}-c_{22} c_{36}+c_{34} c_{36}+c_{26} c_{43}-c_{36} c_{45}-c_{23} c_{46}+c_{35} c_{46}
\end{aligned}
$$

Altogether, since $\operatorname{dim}_{\mathbb{Q}}\left(\operatorname{Lin}_{\mathfrak{M}}\left(I\left(\mathbb{B}_{\mathcal{O}}\right)\right)\right)=6=\# Z$, we apply Corollary 4.2 and conclude that $\operatorname{edim}\left(B_{\mathcal{O}}\right)=18$, that $\Phi$ yields an optimal embedding of the 12 dimensional scheme $\mathbb{B}_{\mathcal{O}}$ into $\mathbb{A}_{\mathbb{Q}}^{18}$, and that the vanishing ideal of the image is minimally generated by 15 quadrics.

\section{ACKNOWLEDGEMENTS}

The second and third authors thank the University of Passau for its hospitality during part of the preparation of this paper. The first and second authors were partially supported by the Vietnam National Foundation for Science and Technology Development (NAFOSTED) grant number 101.04-2019.07.

\section{REFERENCES}

[1] J. Abbott, A.M. Bigatti, and L. Robbiano, CoCoA: a system for doing Computations in Commutative Algebra, available at http://cocoa.dima.unige.it

[2] C. Bertone and F. Cioffi, The close relation between border and Pommaret marked bases, available at arxiv:math/2003.14218 [math.AG].

[3] A.J. Crachiola, The hypersurface $x+x^{2} y+z^{2}+t^{3}$ over a field of arbitrary characteristic, Proc. Amer. Math. Soc. 134 (2005), 1289-1298.

[4] G. Ferrarese and M. Roggero, Homogeneous varieties for Hilbert schemes, Int. J. Algebra 3 (2009), 547-557.

[5] M. Huibregtse, A description of certain affine open schemes that form an open covering of Hilb $_{\mathbb{A}_{2}^{k}}^{n}$, Pacific J. Math. 204 (2002), 97-143.

[6] M. Huibregtse, The cotangent space at a monomial ideal of the Hilbert scheme of points of an affine space, preprint 2005, available at arxiv:math/0506575 [math.AG]

[7] M. Kreuzer, L.N. Long, and L. Robbiano, Computing subschemes of the border basis scheme, Int. J. Algebra Comput. 30 (2020), 1671-1716.

[8] M. Kreuzer and L. Robbiano, Computational Commutative Algebra 1, Springer, Heidelberg, 2000.

[9] M. Kreuzer and L. Robbiano, Computational Commutative Algebra 2, Springer, Heidelberg, 2005.

[10] M. Kreuzer and L. Robbiano, Deformations of border bases, Collect. Math. 59 (2008), 275297.

[11] M. Kreuzer and L. Robbiano, The geometry of border bases, J. Pure Appl. Algebra 215 (2011), 2005-2018. 
[12] M. Kreuzer, B. Sipal, and L.N. Long, On the regularity of the monomial point of a border basis scheme, Beitr. Algebra Geom. 61 (2020), 515-532.

[13] T. Mora and L. Robbiano, The Gröbner fan of an ideal, J. Symb. Comput. 6 (1988), 183-208.

[14] P. Lella and M. Roggero, Rational components of Hilbert schemes, Rend. Sem. Mat. Univ. Padova 126 (2011), 11-45.

[15] A. Reeves and B. Sturmfels, A note on polynomial reduction, J. Symb. Comput. 16 (1993), $273-277$.

[16] L. Robbiano, On border basis and Gröbner basis schemes, Collect. Math. 60 (2009), 11-25.

[17] V. Srinivasan, On the embedding dimension of an affine variety, Math. Ann. 289 (1991), $125-132$.

Fakultät Für Informatik und Mathematik, Universität Passau, D-94030 Passau, GerMANY

Email address: Martin.Kreuzer@uni-passau.de

Fakultät für Informatik und Mathematik, Universität Passau, D-94030 Passau, Germany and Department of Mathematics, University of Education, Hue University, 34 Le Loi Street, Hue City, Vietnam

Email address: lelong@hueuni.edu.vn

Dipartimento di Matematica, Università di Genova, Via Dodecaneso 35, I-16146 GenOVA, ITALY

Email address: lorobbiano@gmail.com 